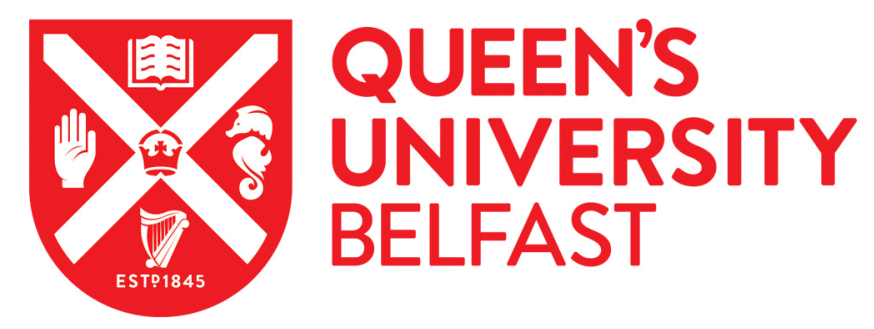

\title{
Pan-STARRS 1 Observations of the Unusual Active Centaur P/2011 S1(Gibbs)
}

Lin, H. W., Chen, Y. T., Lacerda, P., Ip, W. H., Holman, M., Protopapas, P., Chen, W. P., Burgett, W. S.,

Chambers, K. C., Flewelling, H., Huber, M. E., Jedicke, R., Kaiser, N., Magnier, E. A., Metcalfe, N., \& Price, P. A. (2014). Pan-STARRS 1 Observations of the Unusual Active Centaur P/2011 S1(Gibbs). Astronomical Journal, 147(5), [114]. https://doi.org/10.1088/0004-6256/147/5/114

Published in:

Astronomical Journal

Document Version:

Publisher's PDF, also known as Version of record

Queen's University Belfast - Research Portal:

Link to publication record in Queen's University Belfast Research Portal

Publisher rights

Copyright 2014. The American Astronomical Society. All rights reserved. Printed in the U.S.A. This work is made available online in accordance with the publisher's policies.

\section{General rights}

Copyright for the publications made accessible via the Queen's University Belfast Research Portal is retained by the author(s) and / or other copyright owners and it is a condition of accessing these publications that users recognise and abide by the legal requirements associated with these rights.

Take down policy

The Research Portal is Queen's institutional repository that provides access to Queen's research output. Every effort has been made to ensure that content in the Research Portal does not infringe any person's rights, or applicable UK laws. If you discover content in the Research Portal that you believe breaches copyright or violates any law, please contact openaccess@qub.ac.uk. 


\title{
PAN-STARRS 1 OBSERVATIONS OF THE UNUSUAL ACTIVE CENTAUR P/2011 S1(GIBBS)
}

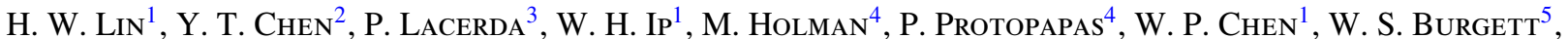

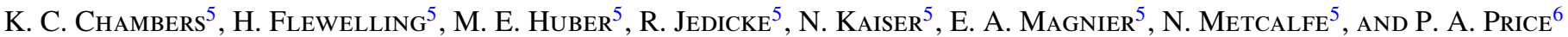 \\ ${ }^{1}$ Institute of Astronomy, National Central University, Taoyuan 32001, Taiwan; edlin@gm.astro.ncu.edu.tw \\ ${ }^{2}$ Institute of Astronomy and Astrophysics, Academia Sinica, P.O. Box 23-141, Taipei 106, Taiwan \\ ${ }^{3}$ Astrophysics Research Centre, School of Mathematics and Physics, Queens University Belfast, Belfast BT7 1NN, UK \\ ${ }^{4}$ Harvard-Smithsonian Center for Astrophysics, 60 Garden Street, Cambridge, MA 02138, USA \\ ${ }^{5}$ Institute for Astronomy, University of Hawaii, 2680 Woodlawn Drive, Honolulu, HI 96822, USA \\ ${ }^{6}$ Department of Astrophysical Sciences, Princeton University, Princeton, NJ 08544, USA \\ Received 2013 August 1; accepted 2014 February 24; published 2014 April 10
}

\begin{abstract}
$\mathrm{P} / 2011 \mathrm{~S} 1$ (Gibbs) is an outer solar system comet or active Centaur with a similar orbit to that of the famous 29P/Schwassmann-Wachmann 1. P/2011 S1 (Gibbs) has been observed by the Pan-STARRS 1 (PS1) sky survey from 2010 to 2012. The resulting data allow us to perform multi-color studies of the nucleus and coma of the comet. Analysis of PS1 images reveals that P/2011 S1 (Gibbs) has a small nucleus $<4 \mathrm{~km}$ radius, with colors $g_{P 1}-r_{P 1}=0.5 \pm 0.02, r_{P 1}-i_{P 1}=0.12 \pm 0.02$, and $i_{P 1}-z_{P 1}=0.46 \pm 0.03$. The comet remained active from 2010 to 2012, with a model-dependent mass-loss rate of $\sim 100 \mathrm{~kg} \mathrm{~s}^{-1}$. The mass-loss rate per unit surface area of P/2011 S1 (Gibbs) is as high as that of 29P/Schwassmann-Wachmann 1, making it one of the most active Centaurs. The mass-loss rate also varies with time from $\sim 40 \mathrm{~kg} \mathrm{~s}^{-1}$ to $150 \mathrm{~kg} \mathrm{~s}^{-1}$. Due to its rather circular orbit, we propose that P/2011 S1 (Gibbs) has 29P/Schwassmann-Wachmann 1-like outbursts that control the outgassing rate. The results indicate that it may have a similar surface composition to that of 29P/Schwassmann-Wachmann 1. Our numerical simulations show that the future orbital evolution of P/2011 S1 (Gibbs) is more similar to that of the main population of Centaurs than to that of $29 \mathrm{P} /$ Schwassmann-Wachmann 1. The results also demonstrate that $\mathrm{P} / 2011 \mathrm{~S} 1$ (Gibbs) is dynamically unstable and can only remain near its current orbit for roughly a thousand years.
\end{abstract}

Key words: comets: general - Kuiper belt objects: individual (Centaur asteroid)

Online-only material: color figures, supplemental data

\section{INTRODUCTION}

The Centaurs are solar system bodies with orbits among four giant planets. For this reason their orbits are unstable, their past and future trajectories are typically chaotic, and their dynamical lifetimes are short (Tiscareno \& Malhotra 2003; Horner et al. 2004). Many theoretical investigations consider this class of object as the transitional population between the Kuiper belt objects and the Jupiter-family comets (JFCs; Fernandez \& Gallardo 1994; Levison \& Duncan 1997; Tiscareno \& Malhotra 2003; Emel'yanenko et al. 2005). The origin of Centaurs is still unclear, but widely accepted sources are the Oort cloud and the scattered disk (Emel'yanenko et al. 2005).

The close relation with the JFCs suggests that some Centaurs may have cometary-like activity. Indeed, the prototype of the Centaurs - (2060) Chiron — has been shown to display cometary activity (Meech \& Belton 1990). Several other "active Centaurs" have been identified (Jewitt 2009). This kind of object prompted searches for evidence of volatile materials. $\mathrm{CN}$ and $\mathrm{CO}$ have been detected in the coma of (2060) Chiron (Bus et al. 1991; Womack \& Stern 1997). Water ice has also been reported on the surface of (2060) Chiron; its detectability is correlated with the level of cometary activity (Luu et al. 2000; Romon-Martin et al. 2003).

29P/Schwassmann-Wachmann 1, which we refer to as $29 \mathrm{P} / \mathrm{SW} 1$, is highly active and is dynamically both a JFC (defined by Tisserand parameter with respect to Jupiter, $T_{J}$, $2<T_{J}<3$; Gladman et al. 2008) and a Centaur (defined by semimajor axis and perihelion, $a_{J}<q<a_{N}$ and $a_{J}<a<a_{N}$, where $a_{J}$ and $a_{N}$ are the semimajor axes of Jupiter and Neptune). Also a Centaur cannot be in a 1:1 mean motion resonance with any planet (Jewitt 2009). Its very circular orbit (eccentricity $\sim 0.04)$, large perihelion distance $(5.72 \mathrm{AU})$, and repeated outburst events set it apart from other comets (Trigo-Rodríguez et al. 2008, 2010). CO has been detected in several studies (Cochran et al. 1982; Senay \& Jewitt 1994; Reach et al. 2013) and is believed to be the source of activity. The outbursts may relate to the nucleus rotation (Trigo-Rodríguez et al. 2010).

In this work, we investigate a new active CentaurP/2011 S1 (Gibbs). P/2011 S1 (Gibbs) was discovered by A. R. Gibbs on 2011 September 18 using the Mt. Lemmon $1.5 \mathrm{~m}$ reflector (Gibbs et al. 2011). JPL classified this object as a Chiron-type comet, which is defined with $T_{J}>3$ and $a>a_{J}$ (Levison \& Duncan 1997). $T_{J}=3.12$ for P/2011 S1 (Gibbs). However, P/2011 S1 (Gibbs) has a small orbital eccentricity $\sim 0.2$, which means it has a quite circular orbit, similar to 29P/SW1. (Lacerda 2013 reported on another similar object, $\mathrm{P} / 2010$ TO20 LINEAR-Grauer (P/LG), as a possible mini $29 \mathrm{P} / \mathrm{SW} 1$ with JPL classification as JFC $\left(2<T_{J}<3\right)$.)

The Panoramic Survey Telescope And Rapid Response System-1 (Pan-STARRS-1, PS1) has serendipitously observed $\mathrm{P} / 2011$ S1 (Gibbs) several times, allowing us to measure astrometry and multi-color photometry at a number of epochs. These data enable comparison of the physical and orbital properties of P/2011 S1 (Gibbs) to those of 29P/SW1 and (2060) Chiron. This work is divided into two main parts. In the first we present the PS1 observations, our photometry, and our analysis of the cometary activity of P/2011 S1 (Gibbs). In the second part we present the results of our numerical integrations, comparing the orbital evolution of $\mathrm{P} / 2011 \mathrm{~S} 1$ (Gibbs) with that of 29P/SW1 and (2060) Chiron. 
Table 1

Observation $\log$ of $\mathrm{P} / 2011 \mathrm{~S} 1$ (Gibbs)

\begin{tabular}{|c|c|c|c|c|c|c|}
\hline Obs Date & Filter & $\begin{array}{c}\text { Number } \\
\text { of exposures }\end{array}$ & $\begin{array}{l}\text { Exp } \\
\text { (s) }\end{array}$ & $\begin{array}{c}\alpha \\
(\operatorname{deg})\end{array}$ & $\begin{array}{c}R \\
(\mathrm{AU})\end{array}$ & $\begin{array}{c}\Delta \\
(\mathrm{AU})\end{array}$ \\
\hline 2010 Sep 29 & $w_{P 1}$ & $45 \mathrm{~s} \times 4$ & 180 & 4.1 & 7.08 & 7.93 \\
\hline 2011 Aug 21 & $g_{P 1}$ & $113 \mathrm{~s} \times 5$ & 565 & 3.5 & 6.64 & 7.56 \\
\hline 2011 Aug 21 & $r_{P 1}$ & $113 \mathrm{~s} \times 4$ & 452 & 3.5 & 6.64 & 7.56 \\
\hline 2011 Aug 23 & $z_{P 1}$ & $240 s \times 6$ & 1440 & 3.2 & 6.63 & 7.56 \\
\hline 2011 Aug 24 & $g_{P 1}$ & $113 \mathrm{~s} \times 6$ & 678 & 3.1 & 6.62 & 7.56 \\
\hline 2011 Aug 24 & $r_{P 1}$ & $113 \mathrm{~s} \times 7$ & 791 & 3.1 & 6.62 & 7.56 \\
\hline 2011 Aug 28 & $i_{P 1}$ & $240 \mathrm{~s} \times 6$ & 1440 & 2.6 & 6.59 & 7.55 \\
\hline 2011 Aug 30 & $g_{P 1}$ & $113 \mathrm{~s} \times 7$ & 791 & 2.3 & 6.58 & 7.55 \\
\hline 2011 Aug 30 & $r_{P 1}$ & $113 \mathrm{~s} \times 5$ & 565 & 2.3 & 6.58 & 7.55 \\
\hline 2011 Aug 31 & $i_{P 1}$ & $240 \mathrm{~s} \times 7$ & 1680 & 2.2 & 6.58 & 7.55 \\
\hline 2011 Sep 1 & $z_{P 1}$ & $240 \mathrm{~s} \times 5$ & 1200 & 2.0 & 6.57 & 7.55 \\
\hline 2011 Sep 2 & $g_{P 1}$ & $113 \mathrm{~s} \times 4$ & 452 & 1.9 & 6.57 & 7.55 \\
\hline 2011 Sep 2 & $r_{P 1}$ & $113 \mathrm{~s} \times 6$ & 678 & 1.9 & 6.57 & 7.55 \\
\hline 2011 Sep 4 & $z P 1$ & $240 \mathrm{~s} \times 4$ & 960 & 1.6 & 6.56 & 7.55 \\
\hline 2011 Sep 4 & $i_{P 1}$ & $45 \mathrm{~s} \times 2$ & 90 & 1.6 & 6.56 & 7.55 \\
\hline 2011 Sep 5 & $g_{P 1}$ & $113 \mathrm{~s} \times 4$ & 452 & 1.5 & 6.55 & 7.54 \\
\hline 2011 Sep 5 & $r_{P 1}$ & $113 \mathrm{~s} \times 7$ & 791 & 1.5 & 6.55 & 7.54 \\
\hline 2011 Sep 7 & $z_{P 1}$ & $240 \mathrm{~s} \times 5$ & 1200 & 1.2 & 6.55 & 7.54 \\
\hline 2011 Sep 8 & $g_{P 1}$ & $113 \mathrm{~s} \times 8$ & 904 & 1.1 & 6.54 & 7.54 \\
\hline 2011 Sep 8 & $r_{P 1}$ & $113 \mathrm{~s} \times 5$ & 565 & 1.1 & 6.54 & 7.54 \\
\hline 2011 Sep 9 & $i_{P 1}$ & $240 \mathrm{~s} \times 7$ & 1680 & 1.0 & 6.54 & 7.54 \\
\hline 2011 Sep 17 & $g_{P 1}$ & $113 \mathrm{~s} \times 8$ & 904 & 0.4 & 6.53 & 7.53 \\
\hline 2011 Sep 17 & $r_{P 1}$ & $113 \mathrm{~s} \times 8$ & 904 & 0.4 & 6.53 & 7.53 \\
\hline 2011 Sep 18 & $i_{P 1}$ & $240 \mathrm{~s} \times 7$ & 1680 & 0.5 & 6.53 & 7.53 \\
\hline 2011 Sep 19 & $z P 1$ & $240 \mathrm{~s} \times 8$ & 1920 & 0.6 & 6.53 & 7.53 \\
\hline 2011 Sep 20 & $g_{P 1}$ & $113 \mathrm{~s} \times 5$ & 565 & 0.7 & 6.53 & 7.53 \\
\hline 2011 Sep 20 & $r_{P 1}$ & $113 \mathrm{~s} \times 6$ & 678 & 0.7 & 6.53 & 7.53 \\
\hline 2011 Sep 21 & $i_{P 1}$ & $240 \mathrm{~s} \times 7$ & 1680 & 0.9 & 6.53 & 7.53 \\
\hline 2012 Oct 9 & $w_{P 1}$ & $45 \mathrm{~s} \times 4$ & 180 & 0.6 & 6.18 & 7.17 \\
\hline 2012 Nov 4 & $w_{P 1}$ & $45 \mathrm{~s} \times 4$ & 180 & 4.2 & 6.30 & 7.15 \\
\hline
\end{tabular}

Note. $\alpha$ is solar phase angle, $R$ is heliocentric distance, and $\Delta$ is geocentric distance.

\section{OBSERVATIONS}

P/2011 S1 (Gibbs) was observed as a part of the PS1 survey. The PS1 telescope is a $1.8 \mathrm{~m}$ Ritchey-Chretien reflector located on Haleakala, Maui, which is equipped with a 1.4 gigapixel camera covering $7 \mathrm{deg}^{2}$ on the sky. The PS1 survey has five different survey modes (Kaiser et al. 2010). (1) The $3 \pi$ steradians survey, which repeatedly covers the $3 \pi$ steradians of sky visible from Haleakala and uses a photometric system that closely approximates the Sloan Digital Sky Survey (SDSS) filter system $g_{P 1}$ (bandpass $\left.\sim 400-550 \mathrm{~nm}\right), r_{P 1}(\sim 550-700 \mathrm{~nm})$, $i_{P 1}(\sim 690-820 \mathrm{~nm}), z_{P 1}(\sim 820-920 \mathrm{~nm})$, and $y_{P 1}(>920 \mathrm{~nm})$ (Tonry et al. 2012). (2) The solar system survey optimized for Near Earth Objects, which concentrates on those ecliptic directions with a wide $\left(w_{P 1}\right)$ band filter that roughly combines the band pass of $g_{P 1}, r_{P 1}, i_{P 1}$ filters. (3) The Medium Deep Survey, which comprises 10 fields spread across the sky and observes nightly with longer exposures in each passband. (4) The Stellar Transit Survey, which searches for Jupiter-like planets in close orbit around stars. (5) A Deep Survey of M31, which studies microlensing and variability in the Andromeda Galaxy.

P/2011 S1 (Gibbs) was observed on Medium-Deep field 10 (MD10), which is centered on the DEEP2-Field 3 Multiwavelength Survey Field, from 2011 August to September, with exposure times of $565 \mathrm{~s}$ to $1980 \mathrm{~s}$ in the $g_{P 1}, r_{P 1}, i_{P 1}$, and $z_{P 1}$ filters. It was also observed in the solar system survey from
Table 2

Improved Orbital Elements of P/2011 S1 (Gibbs)

\begin{tabular}{lc}
\hline \hline Property & Value \\
\hline Semimajor axis, $a$ & $8.6016 \pm 0.0003 \mathrm{AU}$ \\
Eccentricity, $e$ & $0.199602 \pm 8 \times 10^{6}$ \\
Inclination, $i$ & 2.681 \\
Argument of perihelion, $\omega$ & $194.062 \pm 0.008$ \\
Longitude of ascending node, $\Omega$ & $218.897 \pm 0.001$ \\
Next perihelion passage & $2014 \mathrm{Sep} .5$ \\
Perihelion distance, $q$ & $6.8847 \pm 0.0004 \mathrm{AU}$ \\
Aphelion distance, $Q$ & $10.3185 \pm 0.0006 \mathrm{AU}$ \\
\hline
\end{tabular}

Note. The orbital elements solved using the Orbfit code (Bernstein \& Khushalani 2000) with only PS1 detections.

2010 September to 2012 November. It is worth noting that the first PS1 observation was in 2010 May, about one year before $\mathrm{P} / 2011$ S1 (Gibbs) was discovered.

We obtained images of $\mathrm{P} / 2011 \mathrm{~S} 1$ (Gibbs) from the Pan-STARRS postage stamp server. Those images were processed by PS1 image processing pipeline for the image detrending, astrometric solution, and photometry calibration. The "warp" stage images have pixel scale 0 '.25 pixel $^{-1}$ or 0.2 pixel $^{-1}$, depending on which skycell they are located on, and allow the better astrometric solutions for further image stacking. The detailed observation log is shown in Table 1. All available PS1 data are used to improve the orbital solution of P/2011 S1 (Gibbs); the result is shown in Table 2.

\section{COMETARY ACTIVITY}

To detect and trace the existence of cometary activity, we compare the radial profile of $\mathrm{P} / 2011 \mathrm{~S} 1$ (Gibbs) with the local point-spread function (PSF) at every observational epoch.

To maximize the signal-to-noise ratio $(\mathrm{S} / \mathrm{N})$ of images, all of the usable images in each night were median combined in each filter, centered on P/2011 S1 (Gibbs). Another set of stacked images was built from the same postage stamp images but centered on reference stars. The latter were used to build the PSFs for comparison with the target radial profile and photometry calibrations. The final reference PSF was built by averaging roughly a dozen of stars around the target using PSF task of DAOPHOT package in $\mathrm{IRAF}^{7}$.

Four of the P/2011 S1 (Gibbs) stacked images are shown in Figure 1. The two $w_{P 1}$ band filter images are the first and the last observations of P/2011 S1 (Gibbs) taken by Pan-STARRS in the solar system $w_{P 1}$ band survey in 2010 and 2012. The two other images were taken in 2011 as part of MD10 survey. Figure 2 shows the P/2011 S1 (Gibbs) radial profile in four different observational epochs, compared with the reference PSF, plotted with a logarithmic stretch. The radial profiles clearly show a flux excess in outer region when compared with the stellar PSF. This suggests that P/2011 S1 (Gibbs) was continually active from 2010 to 2012. Furthermore, the radial profile of $\mathrm{P} / 2011$ S1 (Gibbs) seems to change in different epochs, hinting that the cometary activity level of this Centaur may have some variation. We further investigate the cometary activity of $\mathrm{P} / 2011$ $\mathrm{S} 1$ (Gibbs) in the next section.

\footnotetext{
7 IRAF is distributed by the National Optical Astronomy Observatory, which is operated by the Association of Universities for Research in Astronomy, Inc., under agreement with the National Science Foundation.
} 


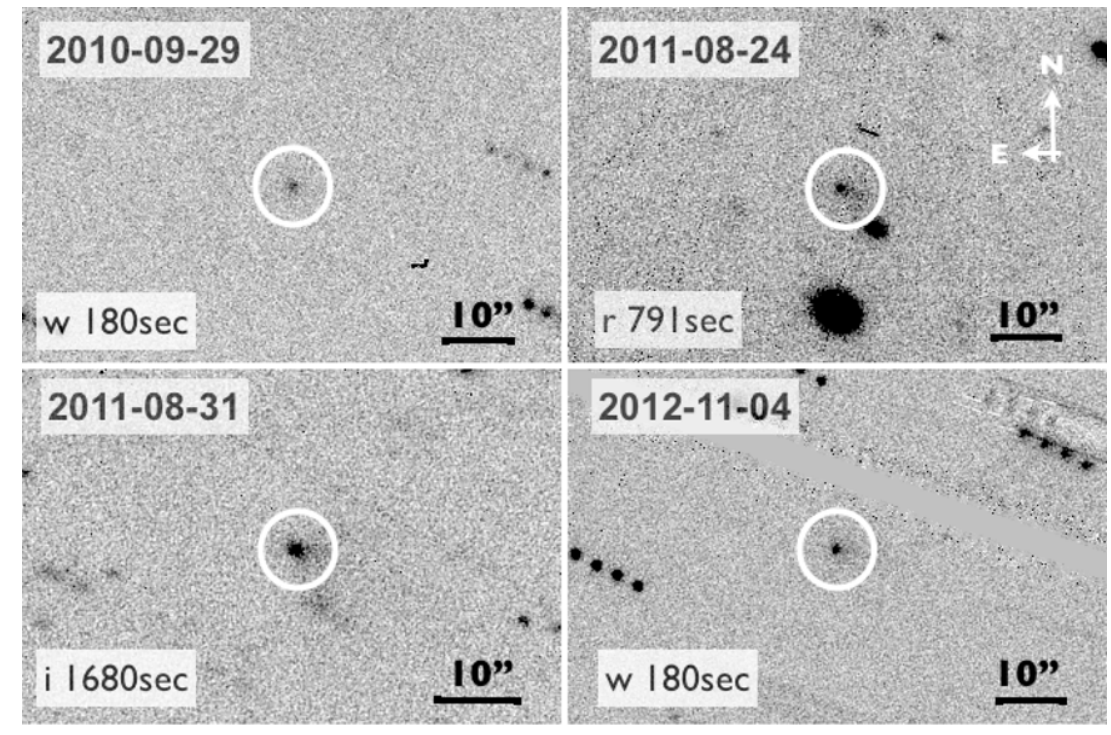

Figure 1. Four P/2011 S1 (Gibbs) stacked images taken by Pan-STARRS 1. The object locates on the center of images and is marked by a white circle. The first image was taken on 2010 September 29, which is one year before the discovery of P/2011 S1 (Gibbs). The second (2011 August 24) and third (2011 August 31 ) images were obtained in the Medium Deep Survey and have a longer exposure time. P/2011 S1 (Gibbs) has a clear coma in these two images. The fourth image was stacked from other set of $w_{P 1}$ band images which were taken in 2012 November. The fuzzy shape shows that P/2011 S1 (Gibbs) continues to be active at that moment.

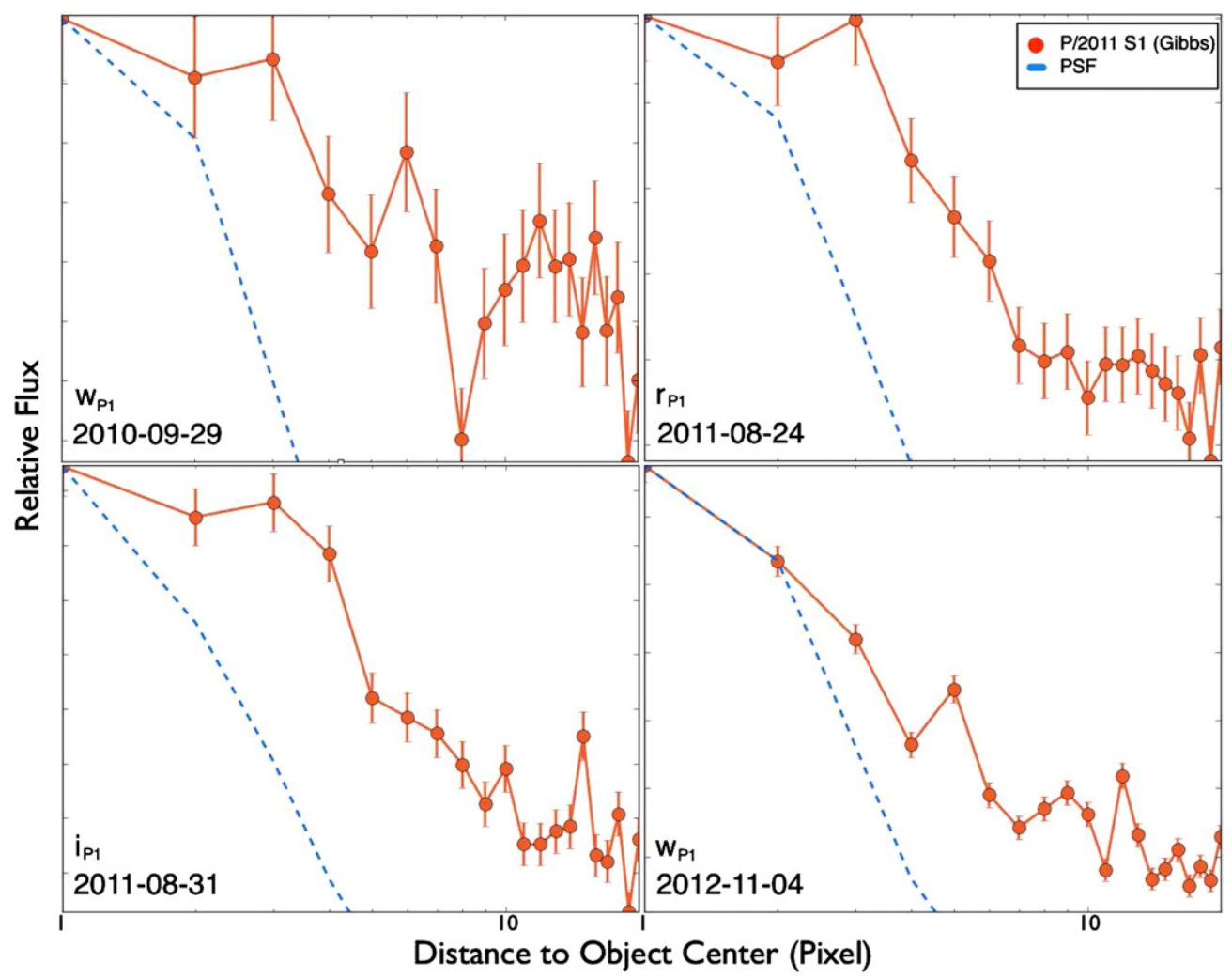

Figure 2. Comparison between the P/2011 S1 (Gibbs) radial profile (red dots) and the reference PSF (dashed blue line) in the four time frames displayed in Figure 1. P/2011 S1 (Gibbs) shows a significant excess in the outer region in comparison with the PSF model, thus indicating the existence of cometary activities.

(A color version of this figure is available in the online journal.)

\section{PHOTOMETRY}

Since the P/2011 S1 (Gibbs) images were taken on different days, the field stars are different. Thus, we are not able to perform differential photometry. To compare the day-to-day brightness variations, we used the PS1 absolute photometry, using the calibrated zero-point of the stacked images.
The stacked reference postage-stamp images, centered on the reference stars (see the previous section), were calibrated by identifying in the field the PS1 catalog stars, which have been calibrated with "uber-calibration" (Schlafly et al. 2012). Uber-calibration is an algorithm to photometrically calibrate wide-field optical imaging surveys, which was first applied on the SDSS imaging data. It can simultaneously solve for 


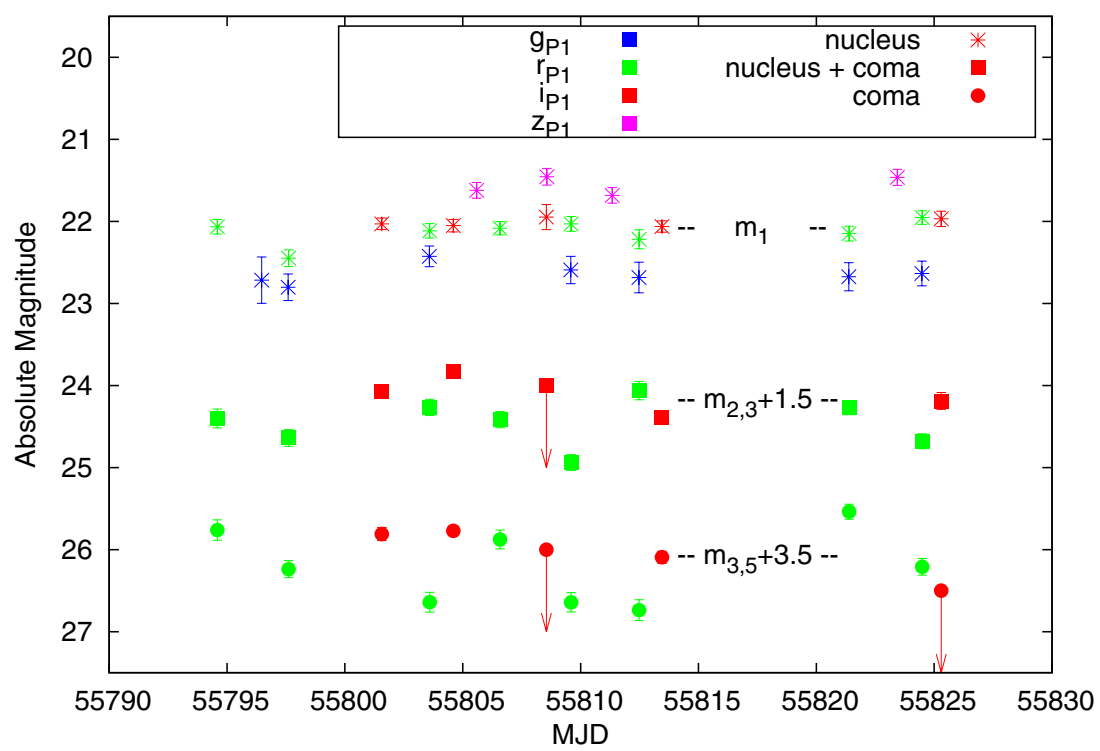

Figure 3. Light curves of P/2011 S1 (Gibbs) in 2011. The star symbols $(*)$ show the central region small aperture (1 FWHM of PSF diameter) measurements $\left(m_{1}\right)$, and refer to the nucleus brightness. The square symbols show the intermediate region annulus ( $2-3$ FWHM of PSF diameter, $m_{2,3}$ ) brightness, containing the flux from both coma and nucleus. The circles show the outer region annulus (3-5 FWHM of PSF diameter, $m_{3,5}$ ) after 9\% of the inner region flux (9\% of $m_{1}$ flux) subtraction, and are the brightness of the pure coma. Some of the measurements without enough flux also show their upper limits in the plot. The light curves in some regions $\left(m_{2,3}\right.$ and $\left.m_{3,5}\right)$ were shifted vertically for clarity of presentation.

(A color version and supplemental data of this figure are available in the online journal.)

the calibration parameters and relative stellar fluxes using overlapping observations (Padmanabhan et al. 2008). Those ubercaled catalogs have a relative precision (compared with SDSS) of $<10 \mathrm{mmag}$ in $g_{P 1}, r_{P 1}$, and $i_{P 1}$, and $\sim 10 \mathrm{mmag}$ in $z_{P 1}$ and $y_{P 1}$ (Schlafly et al. 2012). The reference images share the same zero-point with P/2011 S1 (Gibbs) stack images which are stacked on the center of the object, so that calibration results could be applied to those images. The stars, which have been used to determine the zero points in the reference images, must satisfy the condition that there is no significant brightness variation ( $<0.05$ magnitude) in the first $2 \mathrm{yr}$ of PS 1 observations.

To attempt to isolate the fluxes from the nucleus and the coma, multi-aperture photometry was performed using the PHOT task of the DAOPHOT package in IRAF. This multiaperture photometry does not subtract the sky background for two principal reasons. First, the sky background has already been removed in the PS1 postage stamp image; the background flux is around zero. Second, stellar crowding often prevents an accurate estimate of the background.

We estimate the flux from the cometary nucleus by using a small aperture, and we measure the coma flux by using an outer annulus. We carefully chose the optimal size of aperture and inner/outer radius of the annulus. If the aperture used to measure the nucleus contribution is too large, then it will contain too much flux from the coma. Similarly, if the inner radius of the outer annulus is too small then a significant contribution from the nucleus flux will be included when measuring the coma. Finally, the outer radius of the annulus should not be allowed to extend to regions where the $\mathrm{S} / \mathrm{N}$ from the coma is too small.

Thus, the PSF from the reference images is used to determine which should be the behavior of a point source and therefore of the nucleus only, without the coma. A diameter of 1 FWHM of PSF for the aperture will contain about half of the total flux and is large enough for estimating the flux from the nucleus without including much coma contribution. An annulus with inner and outer diameters of three and five times the FWHM of PSF will only include $9 \%$ of the flux from the Moffat PSF
Table 3

Photometry

\begin{tabular}{lcccc}
\hline \hline Region Measured & $g$ Band & $r$ Band & $i$ Band & $z$ Band \\
\hline$m_{1}$ & $22.51 \pm 0.04$ & $21.99 \pm 0.05$ & $21.87 \pm 0.02$ & $21.42 \pm 0.05$ \\
$m_{2,3}$ & - & $22.96 \pm 0.09$ & $22.62 \pm 0.10$ & - \\
$m_{3,5}$ & - & $22.71 \pm 0.15$ & $22.39 \pm 0.07$ & - \\
\hline
\end{tabular}

and will be suitable for measuring the coma flux. Finally, we decided that two times the flux of 1 FWHM diameter aperture is representative of the nucleus flux, and the coma flux is represented by $91 \%$ of the flux from the annulus with inner and outer diameters of three and five times the FWHM of PSF as the coma flux.

\subsection{Color and Light Curves}

The photometry results and day-by-day brightness variations are shown in Figure 3. The color information could be obtained only from observations acquired in 2011, given that observations were acquired in several filters, while in 2010 and 2012 only one filter $\left(w_{P 1}\right)$ measurements were performed, not allowing us to retrieve any color information. Only the $r_{P 1}$ and $i_{P 1}$ band data have high enough $\mathrm{S} / \mathrm{N}$ to permit photometry of the coma. The brightness variation of the coma region is significantly larger than that of the nucleus region, at least in the $r_{P 1}$ filter (see Figure 3). This implies that the cometary activity is changing with time. We took the average of the measurements to decrease the influence of rotation. We find colors $g_{P 1}-r_{P 1}=$ $0.52 \pm 0.06, r_{P 1}-i_{P 1}=0.12 \pm 0.05, i_{P 1}-z_{P 1}=0.45 \pm 0.05$ for the nucleus region and $r_{P 1}-i_{P 1}=0.32 \pm 0.17$ for coma; the photometry results are shown in Table 3 . These colors are consistent with other active Centaurs, which are found in the blue part $(1.0<B-R<1.4$, for $\mathrm{P} / 2011 \mathrm{~S} 1$ (Gibbs) is $B-R \sim 1.25$, which is calculated using the color transformation equation in Tonry et al. 2012) of the bimodal color distribution of Centaurs (Tegler et al. 2008; Jewitt 2009). 
The coma color seems redder than the nucleus, but it is difficult to make that conclusion because we cannot tell whether the coma is really brighter in the $r_{P 1}$ band or if it comes from the coma brightness variation.

\subsection{Nucleus Size}

The brightness of the nucleus region consists of nucleus flux plus some unknown contribution of coma flux, so the flux can be used to estimate an upper limit of nucleus size. To do so, by assuming that the nucleus has a spherical shape with a crosssection $\pi r^{2}$, the equivalent radius $r$ can be calculated using the relation (Russell 1916):

$$
\pi r^{2} p_{R} 10^{-0.4 \beta \alpha}=2.25 \times 10^{22} \pi R^{2} \Delta^{2} 10^{-0.4\left(m-m_{\odot}\right)},
$$

where $p_{R}$ is the $R$ band geometric albedo which is almost the same as the PS1 $r_{P 1}$ or $i_{P 1}$ band geometric albedo due to the similar band pass. $\beta$ is the linear phase coefficient of the nucleus. $\alpha$ is the solar phase angle, which together with the heliocentric distance, $R$, and the geocentric distance, $\Delta$, can be found in Table 2. The PS1 photometry system apparent magnitude of the Sun at Earth can be converted from the standard photometry system (Tonry et al. 2012). The uncertainty introduced by $\beta$ is negligible when compared to that in $p_{R}$ (uncertain by a factor two or more), if the estimation of nucleus size used the images taken near opposition $\left(\alpha<3^{\circ}\right)$. We assumed $\beta=0.02 \mathrm{mag} \mathrm{deg}^{-1}$ (Millis et al. 1982; Meech \& Jewitt 1987) and $p_{R}=0.1$ (Kolokolova et al. 2004).

The nucleus size estimates obtained separately from high $\mathrm{S} / \mathrm{N} r_{P 1}$ and $i_{P 1}$ band images are consistent. An upper limit to the radius of the nucleus is between 3.1 and $3.9 \mathrm{~km}$.

\subsection{Mass-loss Rate}

We also use the photometry to calculate the coma particle cross-section. Along with several assumptions of coma particle properties, we can estimate the total dust mass present within a coma-dominated annulus. Thus, the mass loss rate can be computed by dividing the total dust mass by the time it takes the dust to move across the annulus. This model-dependent massloss rate is a good indicator to quantify how active is $\mathrm{P} / 2011 \mathrm{~S} 1$ (Gibbs) in comparison to other Centaurs.

First, we take an equivalent dust particle radius of $r_{d}=$ $(0.1 \mu \mathrm{m} \times 1 \mathrm{~cm})^{1 / 2} \sim 32 \mu \mathrm{m}$ which is based on a power-law dust size of $d n / d r_{d} \propto r_{d}^{-3.5}$ with minimum and maximum grain radii of $0.1 \mu \mathrm{m}$ and $1 \mathrm{~cm}$ (Jewitt 2009; Li et al. 2011; Lacerda 2013). Second, the bulk density $\rho_{d}$ is assumed as $1000 \mathrm{~kg} \mathrm{~m}^{-3}$. Third, the total dust cross-section within the annulus, $A_{d}$, can be calculated from Equation (1). Assuming that the particle number density in the coma region is very low, the column number density of particle is $<1$, then the total dust mass within the annulus is

$$
M_{\text {total }}=(4 / 3) \rho_{d} r_{d} A_{d} .
$$

Finally, we need to assume the speed with which the dust is crossing the coma annulus. The velocity $v_{d}$ is highly uncertain and depends on the grain size (Crifo et al. 2004). Estimates based on macroscopic fragment ejection from 17P/Holmes (Stevenson et al. 2010), on the coma expansion velocities of 17P/Holmes (Montalto et al. 2008; Hsieh et al. 2010) and C/Hale-Bopp (Biver et al. 2002), and the spiral jet expansion velocity of 29P/SW1 (Reach et al. 2013), vary from a few $100 \mathrm{~m} \mathrm{~s}^{-1}$ to $1000 \mathrm{~m} \mathrm{~s}^{-1}$. The present work uses $v_{d}=500 \mathrm{~m} \mathrm{~s}^{-1}$. The width of the outer region annulus is equal to the width of



Figure 4. Mass-loss rate variation of $\mathrm{P} / 2011 \mathrm{~S} 1$ (Gibbs) as a function of time. The mass-loss rates were calculated from the results of $r_{p 1}$ photometry (green solid circles) and $i_{p 1}$ photometry (red open circles). The uncertainty of mass-loss rates came from the photometry error.

(A color version and supplemental data of this figure are available in the online journal.)

1 FWHM of PSF. Thus the projected width is dependent on the FWHM of PSF in each image, is between $4000 \mathrm{~km}$ to $8000 \mathrm{~km}$, and results in a dust crossing time from $8000 \mathrm{~s}$ to $16000 \mathrm{~s}$.

Figure 4 shows that the mass-loss rate changes with time. Only $r_{P 1}$ and $i_{P 1}$ band images are used for the best $\mathrm{S} / \mathrm{N}$. Because the orbit is approximately circular, the change in heliocentric distance is small, and the change of heliocentric distance is not the major reason for the variations of mass-loss rate. Also, the mass-loss rate varies from $40 \mathrm{~kg} \mathrm{~s}^{-1}$ to $150 \mathrm{~kg} \mathrm{~s}^{-1}$, suggesting that the distribution of volatility sources may not be uniform over the surface of P/2011 S1 (Gibbs) and small-scale outbursts could take place. Further discussions, together with possible causes for the mass loss, are in Section 6.

\section{DYNAMICAL EVOLUTION}

In this section several questions are addressed. Where did P/2011 S1 (Gibbs) originate? Does it have any relation with other dynamical classes of objects like the Jovian Trojans or Neptune Trojans? Is it dynamically stable? How long can it remain in its current orbit? Is its dynamical evolution similar to that of 29P/SW1 or 2060 Chiron? Or is P/2011 S1 (Gibbs), dynamically, a different type of object?

To answer these questions, we performed a series of numerical orbital integrations to understand the orbital evolution of this object. The orbital elements and covariance matrix were fitted using the Orbfit code (Bernstein \& Khushalani 2000), with only data from PS1 detections. The PS1 observations cover more than $2 \mathrm{yr}$ and provide better astrometric accuracy than other observations. The resulting uncertainties are an order of magnitude smaller than those reported by JPL. We use the N-body integration package Mercury 6.2 (Chambers 1999) to integrate the orbits of 200 massless clones plus P/2011 S1 (Gibbs) with orbital elements as obtained from PS1 observations (Table 2). The clones' orbital elements are assumed normally distributed around the PS1 solution for P/2011 S1 (Gibbs). The calculation is stopped when the semimajor axis exceeds $1000 \mathrm{AU}$. The maximum integration time is 100 million years with an 8 day time step, although only very few of the clones survive the full integration. A high-resolution integration with 

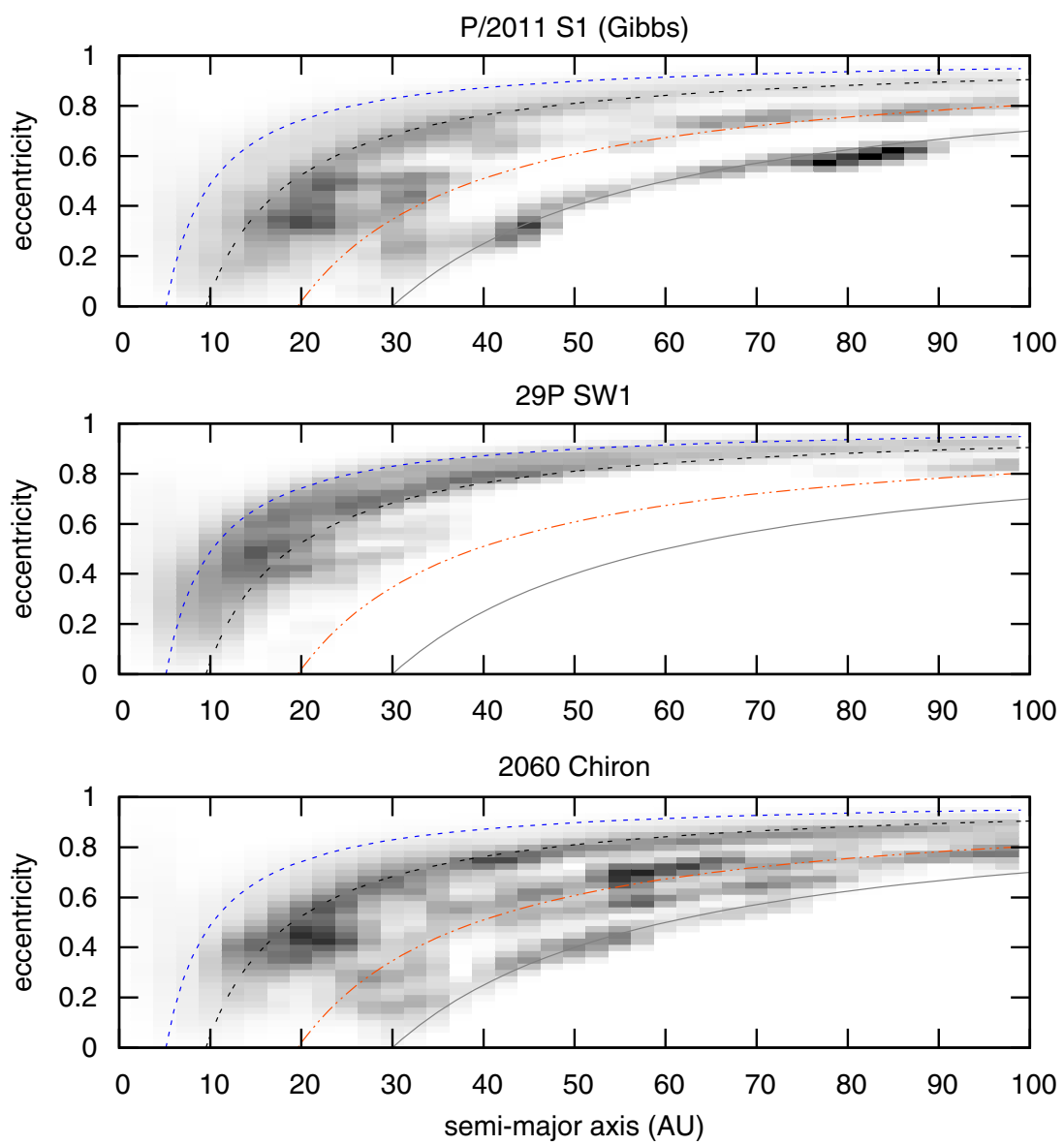

Figure 5. Occupation density map of the dynamical future of P/2011 S1 (Gibbs), 29P/SW 1, and 2060 Chrion. Darker patches have been occupied for a longer time by clones. Dashed lines mark the perihelia of four outer planets.

(A color version of this figure is available in the online journal.)

the Bulirsch-Stoer method with a 1 day time step for $5000 \mathrm{yr}$ is also performed to understand the dynamical evolution of the present orbit. In all of the integrations, we only consider the gravitational force from the Sun and planets. Non-gravitational effects, i.e., out-gassing of object, are omitted.

The integration result shows that the orbit of $\mathrm{P} / 2011 \mathrm{~S} 1$ (Gibbs) is evolving chaotically; every clone has it own evolution path and is not able to trace the precise evolution path of $\mathrm{P} / 2011 \mathrm{~S} 1$ (Gibbs) for a long time ( $<1000 \mathrm{yr})$. Figure 5 shows the dynamical evolution plotted as an occupation density map to present the results statistically.

\subsection{Dynamical Similarity with 29P/Schwassmann- Wachmann 1 and (2060) Chiron}

29P/SW1 and (2060) Chiron (hereafter 2060), two wellknown active Centaurs, have different orbital elements. 29P/SW1 currently has a circular orbit without any planet crossing, but 2060 has a more eccentric orbit between $8 \mathrm{AU}$ and 19 AU within the orbits of Jupiter and Neptune. P/2011 S1 has a rather circular but Saturn-crossing orbit. How do the dynamical behaviors of these objects compare? We study 200 clones for 29P/SW1 and 2060 each in the same way as P/2011 S1 (Gibbs) but with JPL orbital elements to investigate their orbital evolutions, and the results are also shown as an occupation density map in Figure 5. Forward integration results show that 29P/SW1 is more strongly influenced by Jupiter and Saturn; most of time the perihelion of $29 \mathrm{P} / \mathrm{SW} 1$ varies between the semimajor axes of Jupiter and Saturn. In all cases, 29P/SW1 is scattered into an unstable, high eccentric orbit by these two gas giants. The dynamical lifetime is significantly shorter than the lifetimes of the other two Centaurs. (2060) Chiron has a different dynamical trend; the perihelion shifts among the orbits of all of the four outer planets and can be scattered by any one of them. Inspection of the occupation density maps in Figure 5 indicates that the dynamical evolution of $\mathrm{P} / 2011 \mathrm{~S} 1$ (Gibbs) closely resembles that of 2060 Chiron; P/2011 S1 (Gibbs) could also be scattered by any of the four planets, and its dynamical lifetime is longer than that of $29 \mathrm{P} / \mathrm{SW} 1$. Furthermore, looking at the Tisserand parameter with respect to Jupiter, $T_{J}$, of these objects, $29 \mathrm{P} / \mathrm{SW} 1$ has a small $T_{J}(2.984)$, below 3 , meaning that it can be classified as a member of the JFCs, whereas 2060 Chiron and $\mathrm{P} / 2011 \mathrm{~S} 1$ (Gibbs) have larger $T_{J}$ values (3.355 for 2060, 3.122 of P/2011 S1 (Gibbs)) which explains why they are less influenced by Jupiter.

\subsection{Lifetime for Current Near Resonance Orbit with Saturn}

The high-resolution integration shows that the current orbit of $\mathrm{P} / 2011 \mathrm{~S} 1$ (Gibbs) is currently near the 6:5 orbital resonance with Saturn. It may remain near this quasi-resonance orbit for about a thousand years. During that time, P/2011 S1 (Gibbs) has several close encounters with Saturn, and the subsequent orbital evolution path of $\mathrm{P} / 2011 \mathrm{~S} 1$ (Gibbs) becomes too chaotic to trace reliably. 


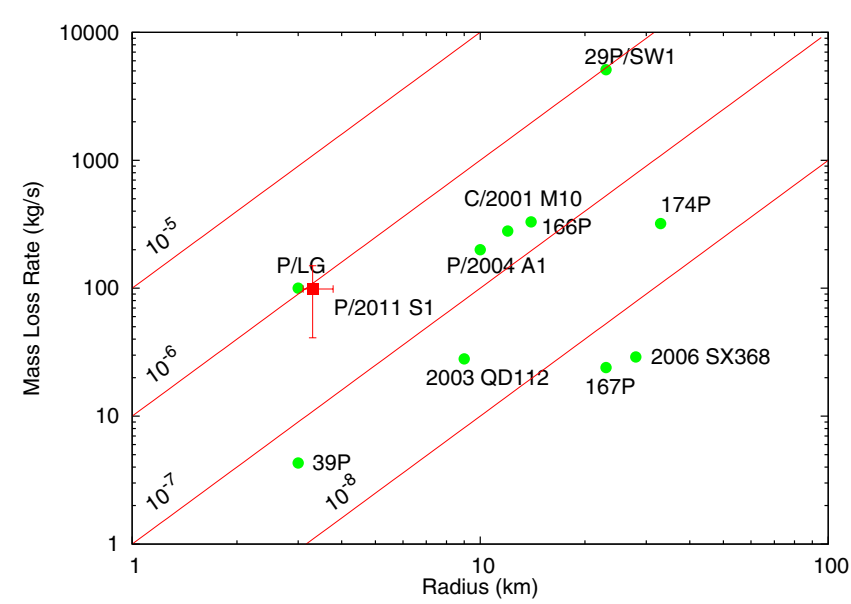

Figure 6. Mass-loss rate vs. nucleus size. The data on P/2011 S1 (Gibbs) are from this work. P/LG is from Lacerda (2013) and others from Jewitt (2009). All of the radius estimations are the upper limit due to the unknown contribution of coma. The solid lines show the specific mass-loss rate and are labeled in units of $\mathrm{kg} \mathrm{m}^{-2} \mathrm{~s}^{-1}$. Notice that except 166P and P/2011 S1 (Gibbs), other objects with a mass-loss rate/radius $>10^{-7} \mathrm{~kg} \mathrm{~s}^{-1} \mathrm{~km}$ have a JFC like Tisserand Parameter $\left(2<T_{J}<3\right)$.

(A color version of this figure is available in the online journal.)

\section{DISCUSSION}

In Section 4 we attributed the variations in coma brightness of $\mathrm{P} / 2011 \mathrm{~S} 1$ (Gibbs) to variations of its mass-loss rate and assume that the size distribution of coma dust particles remains the same. However, we cannot rule out that the changes in coma brightness are due to variations in the coma dust size distribution. A possible scenario that yields a sudden change in the dust particle size distribution is as follows. First, regular outgassing ejects mostly small particles. Later, an outburst ejects the remaining particles that are too large to be lifted by normal outgassing. In this case the mass-loss rate will be larger than our estimation in Section 4.

We use PS1 photometric measurements to estimate the nucleus size and mass-loss rate of P/2011 S1 (Gibbs), and we compare the results with known active Centaurs (see Figure 6). The size and mass-loss rate of 29P/SW1 and P/LG are from Lacerda (2013) and data on the remaining objects are from Jewitt (2009). To evaluate the intrinsic out-gassing activities of different objects, it is better to examine the specific massloss rate, i.e., the mass-loss rate per unit area. If the mass-loss rate is normalized by the upper limit of surface area of the nucleus, a value $10^{-6} \mathrm{~kg} \mathrm{~m}^{-2} \mathrm{~s}^{-1}$ for $\mathrm{P} / 2011 \mathrm{~S} 1$ (Gibbs) is obtained. Thus, similar to 29P/SW1 and P/LG, P/2011 S1 (Gibbs) has a higher mass-loss rate per unit surface area than most other active Centaurs. Note that except for 166P and P/2011 S1 (Gibbs), all other objects with a specific mass-loss rate higher than $10^{-7} \mathrm{~kg} \mathrm{~m}^{-2} \mathrm{~s}^{-1}$ have $T_{J}$ less than 3 and can be classified as a Jovian family comet. The large specific mass-loss rate of Jovian family comets is due to their smaller heliocentric distances compared with that of Centaurs.

The two major influences on the mass-loss rate are the perihelion distance and the composition of volatile materials. Compared with other active Centaurs, P/2011 S1 (Gibbs) does not have a particularly small perihelion. The composition of near surface volatile materials might, thus, be the main reason for its unusually high mass-loss rate. 29P/SW1 has been observed to display CO/CO+ emission (Cochran et al. 1982; Senay \& Jewitt 1994; Gunnarsson et al. 2002; Paganini et al. 2013). On the other hand, Lacerda (2013) suggested that water ice is the source of activity of P/LG. For P/2011 S1 (Gibbs), the perihelion is larger than P/LG and 29P/SW1, so the water production rate should be much lower. Given its larger perihelion distance we would expect P/2011 S1 (Gibbs) to display a lower specific mass-loss rate than $\mathrm{P} / \mathrm{LG}$ if both are driven by water ice sublimation. For this reason, we therefore propose that the composition of $\mathrm{P} / 2011 \mathrm{~S} 1$ (Gibbs) should be similar to 29P/SW1 with $\mathrm{CO}$ as the major source of cometary activity.

Moreover, the presence of significant variation of mass-loss rate of P/2011 S1 (Gibbs), while the heliocentric distance remained the same (See Table 2), indicates that other mechanisms could affect the outgassing rate. CO-rich "hot spots" on the surface of this Centaur may explain this variability. Once a hot spot is heated by the sunlight, it can suddenly increase the mass-loss rate. Under this kind of scheme, the time variation of the massloss rate could be closely related to the rotation of the nucleus, like 29P/SW1 (Trigo-Rodríguez et al. 2008, 2010). The PS1 data are not able to trace the rotation period and outburst period of P/2011 S1 (Gibbs), if it exists. More observations are needed for further investigation.

The total area of active regions can be estimated from the mass-loss rate. Assuming the dust-to-gas mass ratio is $\sim 0.1$ to 1 (Singh et al. 1992; Sanzovo et al. 1996; Kawakita et al. 1997), and the specific mass-loss rate of $\mathrm{CO}$ in $7.5 \mathrm{AU}, 10^{-3} \mathrm{~kg} \mathrm{~m}^{-2} \mathrm{~s}^{-1}$ (Jewitt 2009), the active region is around $0.1-1 \%$ of the total surface area of P/2011 S1 (Gibbs). The result is also consistent with the assumption of the existence of active hot spots.

Assuming a $100 \mathrm{~kg} \mathrm{~s}^{-1}$ mass loss rate and $0.1-1 \%$ active surface area, we can estimate the active area recession rate of P/2011 S1 (Gibbs) to be $\sim 0.3-3 \mathrm{~km}$ per thousand years. Considering that the lifetime of $\mathrm{P} / 2011 \mathrm{~S} 1$ (Gibbs) around the current orbit is only about a thousand years, this object cannot always remain active; this activity event must be recent. A possible explanation is that the hot spots of $\mathrm{P} / 2011 \mathrm{~S} 1$ (Gibbs) were produced by some recent impacts or other mechanisms, uncovering volatile $\mathrm{CO}$ ice. Once the $\mathrm{CO}$ hot spots have been covered by dust mantle or $\mathrm{CO}$ runs out, the activity of P/2011 S1 (Gibbs) will soon stop.

The orbital integration results suggest that the future of $\mathrm{P} / 2011 \mathrm{~S} 1$ (Gibbs) is closer to the main population of Centaurs than 29P/SW1-like objects. We are led to believe that dynamically 29P/SW1 and P/2011 S1 (Gibbs) may represent an intermediate stage between Centaurs and JFCs, with 29P/SW1 closer to the JFCs and P/2011 S1 (Gibbs) closer to Centaurs.

\section{CONCLUSIONS}

We report photometric observations of active Centaur P/2011 S1 (Gibbs), improved orbital elements obtained from PS1 survey images, and numerical simulations of its orbital evolution. Our results can be summarized as follows.

P/2011 S1 (Gibbs) was active in 2010, one year before the discovery by A. R. Gibbs, and remained active in 2012.

The nucleus of $\mathrm{P} / 2011 \mathrm{~S} 1$ (Gibbs) has a radius $<4 \mathrm{~km}$ and colors $g_{P 1}-r_{P 1}=0.52 \pm 0.06, r_{P 1}-i_{P 1}=0.12 \pm 0.05$, and $i_{P 1}-z_{P 1}=0.45 \pm 0.05$, consistent with other known active Centaurs. The data also show that the coma materials appear significantly redder than the nucleus. The brightness of the coma varies with time, suggesting several small-scale outburst events in the observation period.

The model-dependent mass-loss rate of P/2011 S1 (Gibbs) $\sim 100 \mathrm{~kg} \mathrm{~s}^{-1}$. The mass-loss rate per surface area is higher than other active Centaurs and as high as 29P/SchwassmannWachmann 1. It also varies with time from $\sim 40 \mathrm{~kg} \mathrm{~s}^{-1}$ to 
$150 \mathrm{~kg} \mathrm{~s}^{-1}$. This observed mass-loss rate variation is not related to the heliocentric distance, because the orbit of P/2011 S1 (Gibbs) is rather circular. We propose the occurrence of a 29P/ SW1-like outburst effect but more and long-term observations are needed to test this scenario.

Numerical simulations show that the future orbital evolution of $\mathrm{P} / 2011 \mathrm{~S} 1$ (Gibbs) is more similar to that of the Centaur (2060) Chiron rather than to 29P/Schwassmann-Wachmann 1. The results also show that $\mathrm{P} / 2011 \mathrm{~S} 1$ (Gibbs) is dynamically unstable and can remain near its current orbit for only a thousand years or so.

Finally, given its unusually high mass-loss rate and orbital evolution results, we have come to the conclusion that $\mathrm{P} / 2011$ S1 (Gibbs) has similar near-surface composition to 29P/SW1 but an orbit typical of a Centaur.

We thank for J. J. Kavelaars for his helpful comments on the manuscript. We also acknowledge the anonymous referee's useful suggestions for improving the manuscript.

This work was supported in part by NSC grants NSC 1022119-M-008-001 and NSC 101-2119-M-008-007-MY3 and the Ministry of Education under the 5500 Program NCU.

The Pan-STARRS1 Surveys (PS1) have been made possible through contributions of the Institute for Astronomy, the University of Hawaii, the Pan-STARRS Project Office, the Max-Planck Society and its participating institutes, the Max Planck Institute for Astronomy, Heidelberg, and the Max Planck Institute for Extraterrestrial Physics, Garching, The Johns Hopkins University, Durham University, the University of Edinburgh, Queen's University Belfast, the Harvard-Smithsonian Center for Astrophysics, the Las Cumbres Observatory Global Telescope Network Incorporated, the National Central University of Taiwan, the Space Telescope Science Institute, the National Aeronautics and Space Administration under grant No. NNX08AR22G issued through the Planetary Science Division of the NASA Science Mission Directorate, the National Science Foundation under Grant No. AST-1238877, and the University of Maryland.

\section{REFERENCES}

Bernstein, G., \& Khushalani, B. 2000, AJ, 120, 3323

Biver, N., Bockelée-Morvan, D., Colom, P., et al. 2002, EM\&P, 90, 5

Bus, S. J., A'Hearn, M. F., Schleicher, D. G., \& Bowell, E. 1991, Sci, 251, 774

Chambers, J. E. 1999, MNRAS, 304, 793
Cochran, A. L., Cochran, W. D., \& Barker, E. S. 1982, ApJ, 254, 816

Crifo, J. F., Fulle, M., Kömle, N. I., \& Szego, K. 2004, Comets II, ed. M. C. Festou, H. U. Keller, \& H. A. Weaver (Tucson, AZ: Univ. Arizona Press), 471

Emel'yanenko, V. V., Asher, D. J., \& Bailey, M. E. 2005, MNRAS, 361, 1345

Fernandez, J. A., \& Gallardo, T. 1994, A\&A, 281, 911

Gibbs, A. R., Tornero, S. F., \& Williams, G. V. 2011, IAUC, 9234, 1

Gladman, B., Marsden, B. G., \& Vanlaerhoven, C. 2008, The Solar System Beyond Neptune, ed. M. A. Barucci et al. (Tucson, AZ: Univ. Arizona Press), 43

Gunnarsson, M., Rickman, H., Festou, M. C., Winnberg, A., \& Tancredi, G. 2002, Icar, 157, 309

Horner, J., Evans, N. W., \& Bailey, M. E. 2004, MNRAS, 354, 798

Hsieh, H. H., Fitzsimmons, A., Joshi, Y., Christian, D., \& Pollacco, D. L. 2010, MNRAS, 407, 1784

Jewitt, D. 2009, AJ, 137, 4296

Kaiser, N., Burgett, W., Chambers, K., et al. 2010, Proc. SPIE, 7733, 77330

Kawakita, H., Furusho, R., Fujii, M., \& Watanabe, J.-I. 1997, PASJ, 49, L41

Kolokolova, L., Hanner, M. S., Levasseur-Regourd, A.-C., \& Gustafson, B. Å. S. 2004, Comets II, ed. M. C. Festou, H. U. Keller, \& H. A. Weaver (Tucson, AZ: Univ. Arizona Press), 577

Lacerda, P. 2013, MNRAS, 428, 1818

Levison, H. F., \& Duncan, M. J. 1997, Icar, 127, 13

Li, J., Jewitt, D., Clover, J. M., \& Jackson, B. V. 2011, ApJ, 728, 31

Luu, J. X., Jewitt, D. C., \& Trujillo, C. 2000, ApJL, 531, L151

Meech, K. J., \& Belton, M. J. S. 1990, AJ, 100, 1323

Meech, K. J., \& Jewitt, D. C. 1987, A\&A, 187, 585

Millis, R. L., Ahearn, M. F., \& Thompson, D. T. 1982, AJ, 87, 1310

Montalto, M., Riffeser, A., Hopp, U., Wilke, S., \& Carraro, G. 2008, A\&A, 479, L45

Padmanabhan, N., Schlegel, D. J., Finkbeiner, D. P., et al. 2008, ApJ, 674, 1217

Paganini, L., Mumma, M. J., Boehnhardt, H., et al. 2013, ApJ, 766, 100

Reach, W. T., Kelley, M. S., \& Vaubaillon, J. 2013, Icar, 226, 777

Romon-Martin, J., Delahodde, C., Barucci, M. A., de Bergh, C., \& Peixinho, N. 2003, A\&A, 400, 369

Russell, H. N. 1916, ApJ, 43, 173

Sanzovo, G. C., Singh, P. D., \& Huebner, W. F. 1996, A\&AS, 120, 301

Schlafly, E. F., Finkbeiner, D. P., Jurić, M., et al. 2012, ApJ, 756, 158

Senay, M. C., \& Jewitt, D. 1994, Natur, 371, 229

Singh, P. D., de Almeida, A. A., \& Huebner, W. F. 1992, AJ, 104, 848

Stevenson, R., Kleyna, J., \& Jewitt, D. 2010, AJ, 139, 2230

Tegler, S. C., Bauer, J. M., Romanishin, W., \& Peixinho, N. 2008, The Solar System Beyond Neptune, ed. M. A. Barucci et al. (Tucson, AZ: Univ. Arizona Press), 105

Tiscareno, M. S., \& Malhotra, R. 2003, AJ, 126, 3122

Tonry, J. L., Stubbs, C. W., Lykke, K. R., et al. 2012, ApJ, 750, 99

Trigo-Rodríguez, J. M., García-Hernández, D. A., Sánchez, A., et al. 2010, MNRAS, 409, 1682

Trigo-Rodríguez, J. M., García-Melendo, E., Davidsson, B. J. R., et al. 2008, A\&A, 485, 599

Womack, M., \& Stern, S. A. 1997, Lunar and Planetary Institute Science Conference Abstracts, 28, 1575 